\title{
Research and Analysis of the Effect of Heat Treatment on Damping Properties of Ductile Iron
}

https://doi.org/10.1515/phys-2019-0058

Received Apr 30, 2019; accepted Jun 14, 2019

\begin{abstract}
With the continuous development of metal manufacturing technology, high-strength and high-hardness ductile iron materials have excellent comprehensive performance. Many performance indexes are comparable to those of alloy steels, and they have excellent casting properties. Many large-scale parts produced by cast steel are slowly being replaced by this material. Ductile iron is obtained by a spheroidizing treatment and inoculation to obtain spheroidal graphite. The mechanical properties of cast iron have been effectively improved, especially plasticity and toughness, and the strength obtained is higher than that of carbon steel. Ductile iron has the properties of iron and the properties of steel. It is a new type of engineering material with high plasticity, strength, corrosion resistance, and wear-resistance. Because of its excellent performance, it has been successfully used to cast parts with high-stress conditions, high strength, toughness and wear resistance. Due to the small splitting effect of ductile iron on the metal matrix, the stress concentration is effectively eliminated. Therefore, the matrix structure of ductile cast iron is changed by heat treatment, thereby improving its mechanical properties and the damping performance of the material itself. Through a heat treatment process experiment of ductile iron, the related process and technical measures of damping performance in the heat treatment production process are obtained.
\end{abstract}

Keywords: alloy ductile iron; piston ring; heat treatment; deformation

PACS: 46.40.Ff, 44.10.+i

\footnotetext{
*Corresponding Author: Yu Zhang: School of Materials' Science and Technology, Harbin University of Science and Technology, Harbin 150000, China; Institute of Advanced Technology, Heilongjiang Academy of Sciences, Harbin 150000, China; Email: newstar1314@126.com Erjun Guo, Liping Wang, Yicheng Feng, Sicong Zhao: School of Materials' Science and Technology, Harbin University of Science and Technology, Harbin 150000, China Meihui Song: Institute of Advanced Technology, Heilongjiang Academy of Sciences, Harbin 150000, China
}

ə Open Access. ๑ 2019 Y. Zhang et al., published by De Gruyter. License

\section{Introduction}

In recent years, the cast iron metallurgy industry has developed rapidly, especially the heat treatment technology of ductile iron. It is a new material product which is not only low in cost but also better to craft. Its high strength is an essential part of the cast iron metallurgy industry, and its broad application prospects have made it a highly anticipated material. Based on this, the study of the heat treatment process of ductile iron conforms to the development trend [1], and it is hoped that the development of heat treatment technology will be promoted through research. Damping performance is a critical factor in the heat treatment of ductile iron, and it is essential to pay attention to this aspect of the technology, which is related to the quality of the heat treatment process. In the casting process, the internal and external cooling speeds of the cast iron parts are not the same, and it si easy to form internal stresses. If these are not eliminated in time, it is easy to crack the parts during use or cutting, which affects the quality of the product [2].

\section{Damping characteristics analysis of ductile iron materials}

The damping of a ductile iron material is a timedependent, elastic-related physical property [3]. For an ideal elastomer, the relationship between the stress applied to the material and the induced strain under external force follows Hooke's law, that is, the strain $\epsilon$ is proportional to the stress $\sigma$, and E represents Young's modulus.

$$
\sigma=E \cdot \varepsilon
$$

The relationship between the stress and strain of an ideal elastomer should satisfy the three conditional linear relationships: the response of the strain to each stress is linear, this response is instantaneous, i.e., the strain and stress are always in phase, and each strain is only in response to its corresponding applied stress [4]. 
Table 1: Metal Additives in Heat Treatment of Ductile Iron

\begin{tabular}{cccccccc}
\hline Chemical element & $\mathrm{Sn}$ & $\mathrm{Mo}$ & $\mathrm{Cu}$ & $\mathrm{Ti}$ & $\mathrm{Mn}$ & $\mathrm{Ni}$ & $\mathrm{Cr}$ \\
Proportion in ductile iron & 40 & 8.1 & 5.1 & 4.5 & 0.45 & 0.45 & 0.34 \\
\hline
\end{tabular}

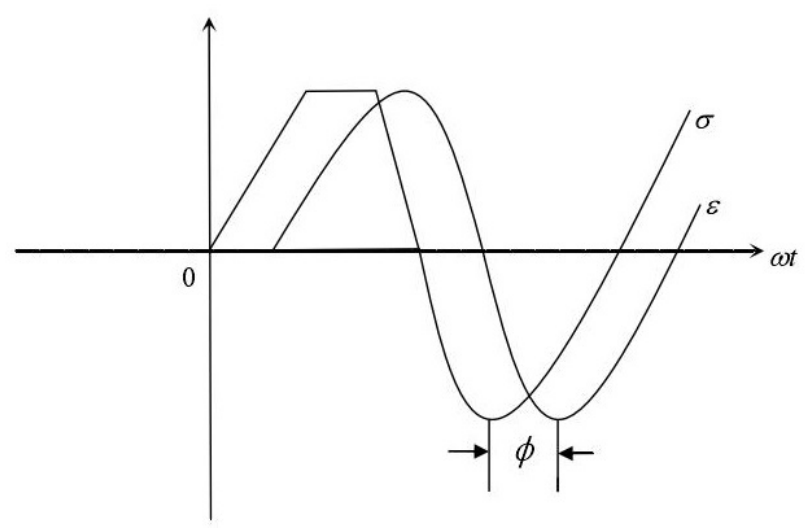

Figure 1: Hysteresis relationship between strain and stress of spheroidal graphite cast iron under stress

For actual materials, stress and strain often cannot meet the above three conditions at the same time, resulting in inelastic behavior. The response of the material to the applied stress is not only a time-independent transient elastic strain, but also a time-dependent strain that lags behind the applied load [5], whereas for actual materials, inelastic behavior is usually produced, i.e. the strain lags behind the loaded stress [6], as shown in Figure 1.

\subsection{Metal additive in the heat treatment process of ductile iron}

\subsubsection{Alloying elements}

Alloying elements are different from the essential constituent elements of cast iron materials and essential elements in spheroidizing agents. The relative price of alloying elements is relatively high. The proper amount of alloy plays an essential role in ductile iron so it should be analyzed separately [7]. Each alloying element provides different properties to the ductile iron: Sn promotes the strongest nodular cast iron compared to $\mathrm{Cr}$ which is relatively weak. The effects and applicable conditions of alloying elements such as $\mathrm{Cu}, \mathrm{Sn}, \mathrm{Cr}, \mathrm{Mn}, \mathrm{Ni}$, and Mo are individually analyzed below, and their proportions are presented in Table 1.

\subsubsection{Copper element}

Copper is an essential alloying element and is often added to cast iron. Copper can promote the role of graphitization, which can reduce or eliminate the formation of free cementite. In the eutectoid transformation, it is beneficial to the formation of a spheroidal graphite cast iron matrix, which can reduce or inhibit the formation of ferrite. It improves the hardenability of thick-walled parts, improves the uniformity of cross-section structure and performance, and is negatively segregated and enriched inside the eutectic group.

With the increase of the proportion of copper added, the strength and hardness of the material will be correspondingly enhanced. In terms of elongation after fracture, the effect on the ductile iron base is not apparent [8].

In general, the price of copper is relatively moderate, and it is a relatively mild alloying element. The addition of copper is beneficial for the properties of casting materials, and the side effects are small. However, the amount of copper added is generally controlled within $2 \%$ to avoid the occurrence of adverse effects on the spheroidization of the copper-rich phase or interfering elements in the copper. As the alloying element, the necessary control of copper is also required [9].

\subsubsection{Molybdenum}

Molybdenum is also an essential alloying element which can improve the hardenability of thick-walled parts. It does not affect the production and spheroidization of graphite spheres. It can refine the eutectic group, which is positively segregated and enriched at the eutectic boundary [10].

Molybdenum can play a role in improving strength and is more evident than manganese and copper [11]; however, the elongation at break and the impact toughness decrease as the strength increases. For as-cast nodular cast iron ductile iron, the elongation at break and the impact toughness decrease are small, while the increase in yield strength is more significant than the increase in tensile strength, thus effectively improving the yield ratio [12].

Molybdenum is a typical carbide-forming element and promotes the formation of carbides significantly stronger 
than manganese and Cr. It is a strong catalyst for the production of ductile iron. When the molybdenum content is less than $0.6 \%$, the effect is mild and slightly promotes white mouth. When the molybdenum content exceeds $0.6 \%$, a large amount of carbide containing molybdenum appears at the boundary of the eutectic group [13]. This carbide is stable and difficult to decompose after prolonged high-temperature degradation. Therefore, the amount of molybdenum should be controlled by $0.6 \%$.

\subsubsection{Nickel}

Nickel is a graphitizing element, which can reduce the white mouth tendency and has no obvious influence on the graphite morphology and the number of eutectic groups. Like copper, nickel is negatively segregated in the solidification of ductile iron and rich in primary austenite and eutectic [14]. While the residual iron is depleted, the promotion of ductile iron by nickel is not very large. However, nickel can improve the section sensitivity of thick section castings. Therefore, as the nickel content increases, the hardness after normalization increases significantly. In order to increase the hardness of the material, it is feasible to add some nickel properly, and it will have a significant effect [15].

\subsubsection{Chromium}

Adding chromium can produce fine-grained spheroidal graphite cast iron and can improve the hardenability. However, very little chromium can form carbides [16]. The carbide is reticulated and enriched at the boundary of the eutectic. It takes a long time to decompose and promotes the formation of ductile iron. Therefore, chromium content generally cannot exceed $0.5 \%$.

\subsubsection{Manganese}

Manganese can effectively promote the formation of carbide in ductile iron, but the effect is limited. When solidifying ductile iron, manganese can increase the tendency of white mouth. At the same time, for thick-walled parts, the segregation tendency of manganese is particularly serious [17], and the formed carbide is reticulated on the grain boundary, which adversely affects the performance. Therefore, it is suitable to control the content of manganese within $0.6 \%$.

\subsection{Production equipment for heat treatment damping of ductile iron}

Alloy melting furnaces are often used for special alloy casting. Usually, they consist of a power supply, a furnace body, a vacuum system, and a water-cooling system. A power vacuum induction furnace is the same as a medium frequency induction furnace, but it is smelted under vacuum, and its power supply has some special requirements. The low terminal potential of the inductor prevents high-order harmonics from entering the load circuit oscillation circuit.

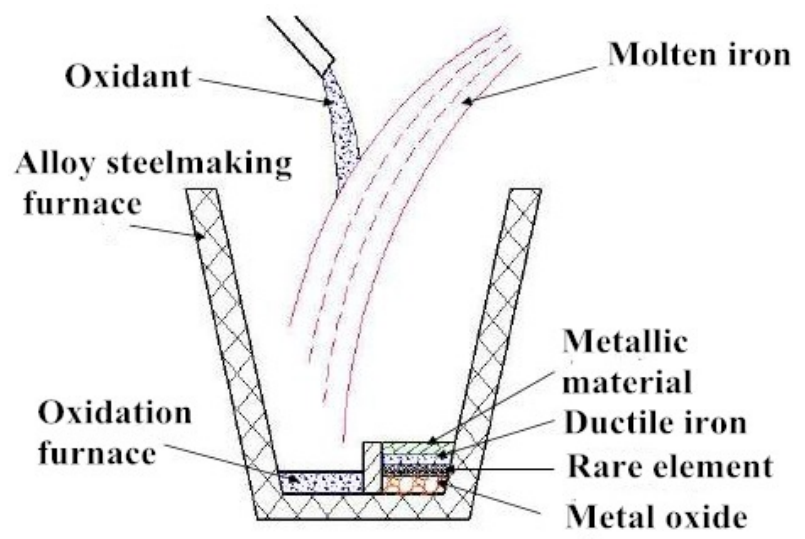

Figure 2: Refining process of metal oxide furnace

The furnace body of a vacuum induction furnace is composed of a furnace shell, an inductor, a tilting furnace mechanism, a casting system, a water-cooling system, and a power transmission device. The most important feature of a vacuum induction furnace is the fact that the smelting and casting process takes place in the furnace shell. The shell must have sufficient structural strength to withstand the strong pressure created by the internal vacuum. The smelting and sample preparation of the damping alloy produces a single ferrite structure. It is an important ferrite forming element, which can improve the corrosion resistance and strength of the alloy. It is also a strong solid solution strengthening element, and its addition can also improve tempering stability. The interstitial atoms affect adversely on the damping properties of the alloy, and the alloy must be strictly controlled during formulation. Industrial pure iron, metal chromium, and metal aluminum are used as raw materials and refined in the above alloy oxidation furnace [18]. 

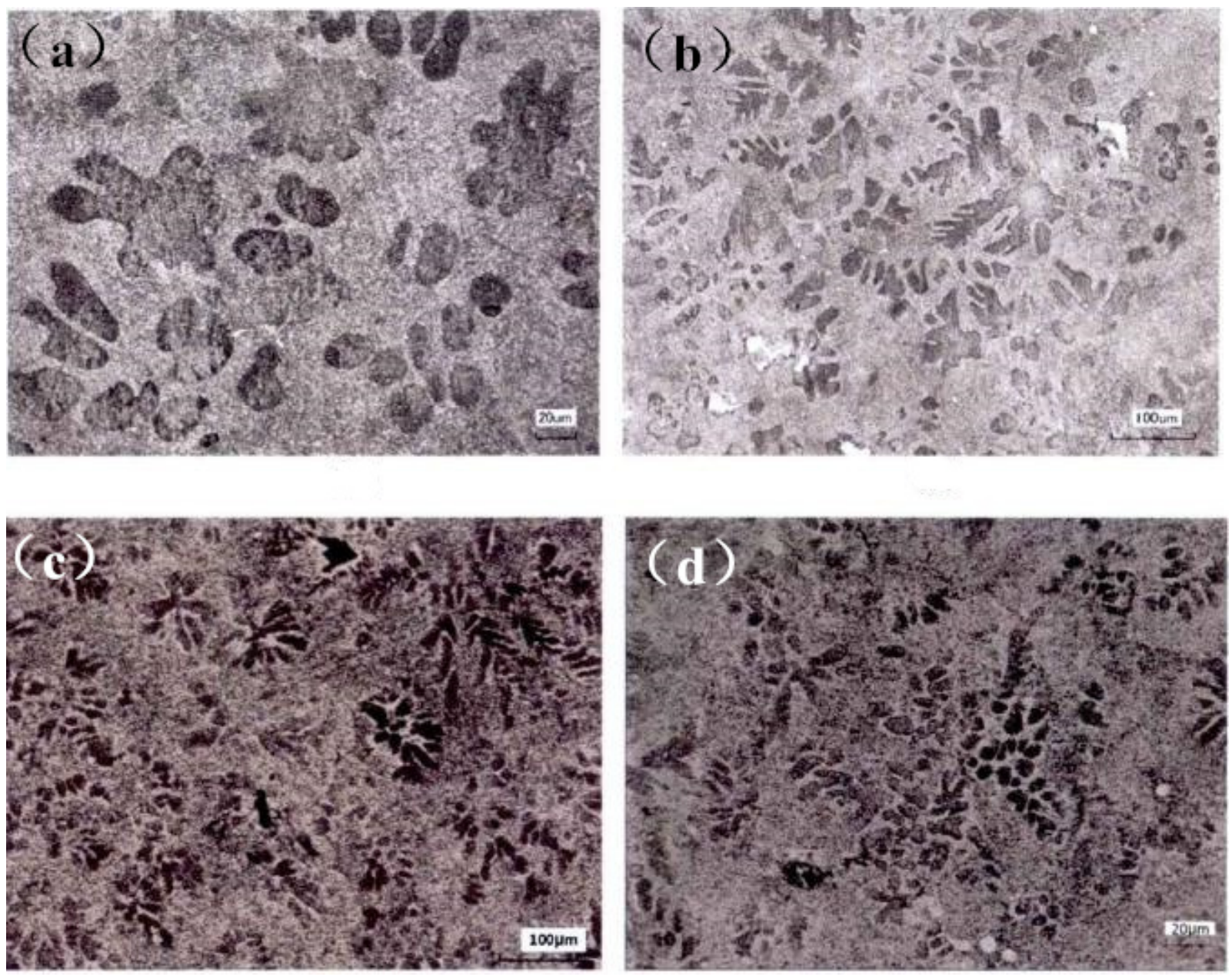

Figure 3: Structure of spheroidal graphite cast iron in different microscope modes

\subsection{Damping test and characterization}

During the free decay process, the vibration amplitude of the sample gradually decreases with time. The damping property of the sample can be calculated from the amplitude attenuation value. In the experimental test and analysis process, the free vibration attenuation curve is often involved, the damping strain curve occupies a curve as does the damping temperature.

\subsection{Structural Characterization of Ductile Iron under Different Treatments}

When observed by electron microscopes with wavelengths of $20 \mu \mathrm{m}$ and $100 \mu \mathrm{m}$ respectively, it can be found in Figure 3(a) and Figure 3(b) that the crystals of ductile iron are large, the overall trend is not much different from the heat treatment, and they all appear as granules. The spheroidal graphite cast iron after heat treatment can be seen from the metallographic microstructure diagram, which effectively improves the dendrite segregation of nodular cast iron. Regardless of the size of the formed crystal grains, it is equiaxed crystal, and the composition is compared with casting. The state of the metal is also gradually uniformized. It can be seen from Figure 3(c) and Figure 3(d) that the grain size produced by the 350-degree heat treatment method is significantly uniform and finer than that of the other two heat treatment modes. In the as-cast state, the gray-black eutectoid is mainly composed of white phase particles. After heat treatment, the eutectoids are transformed, the black phase is precipitated, and the black phase of the grains is dominant. The heat treatment causes the crystal segregation in the alloy to be improved. As the heat treatment time increases, some low-point inclusions in the alloy will decompose, at the same time, the amplitude-modulated decomposition will occur, and the original lamellar and eutectoids will be transformed into granular ones. The heat treatment improves the dendrite 


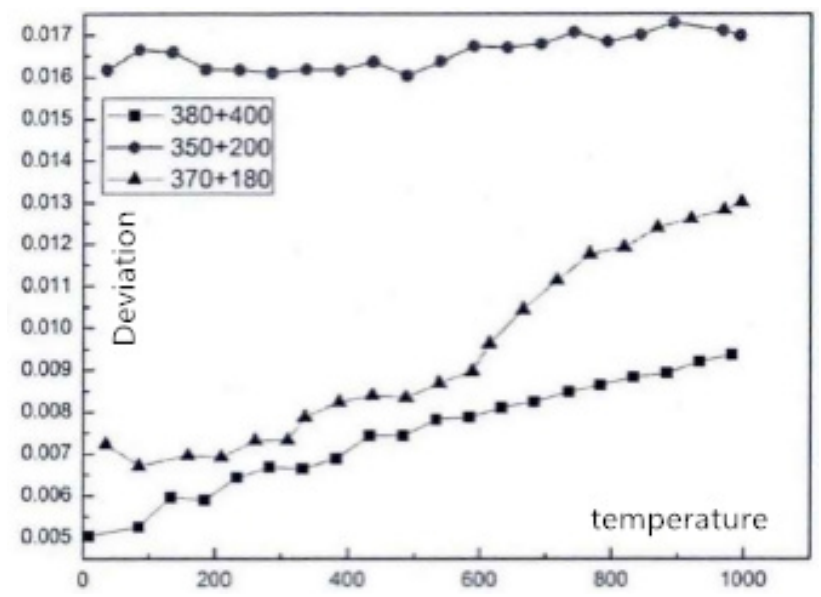

Figure 4: Damping strain amplitude curve of nodular cast iron under different heat treatment modes

segregation in the alloy. The difference is the size of the two aging methods. It is differently shown in Figure 4.

The most obvious difference between these two materials is that the grain refinement is highest after heat treatment. The heat treatment results in improved dendrite segregation and makes the composition more uniform. Since the solidification process in the as-cast state is completed in a non-equilibrium state, many low-burning eutectics are interposed in the grain boundary, which affects the slidability of the interface. The heat treatment can improve the segregation of the components, decompose the lowburning crystallites in the grain boundary, and improve the slidability of the interface. The product of the amplitude modulation decomposition of the alloy after the heat treatment is further refined. The original lamellar phase and the eutectoid phase follow a certain orientation relationship and are arranged in a regular pattern. There is a certain coherent interface relationship between them, and the mobility is not large. After the heat treatment, the lamellar two phases are transformed into the granular phase, the original coherent interface relationship no longer exists, forming a non-coherent interface to greatly increase the mobility of the grain boundary. The heat treatment increases the number of mobile interfaces and the mobility of the interface, and the damping performance of the ductile iron is improved.

As shown in the whole curve, the damping performance between $200-280^{\circ} \mathrm{C}$ is the best, and the difference between the peak value of the curve and the initial measured value exceeds 0.01 . It can be seen that the damping performance of ductile iron is sensitive to the test temperature. The reason why spheroidal graphite casts a peak between $200-280^{\circ} \mathrm{C}$ is the average result of the interaction of various damping mechanisms such as damping of

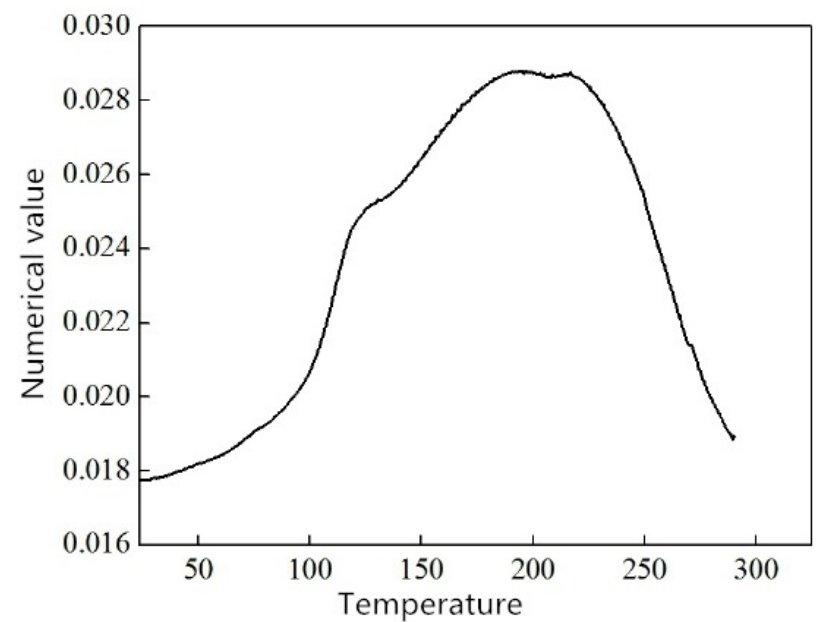

Figure 5: Heat treatment problem and the influence of nodular cast iron damping

ductile cast iron, damping by gap atomic micro-diffusion, and dislocation damping. Since the temperature range of 200- $280^{\circ} \mathrm{C}$ does not cause Magnetic tissue transformation, there is no need to consider the effects of ferromagnetic damping. With the increase of temperature and the interaction between the complex damping mechanisms, the internal friction peak of ductile iron appears between 200$280^{\circ} \mathrm{C}$, which means that the material has excellent damping performance in this operating temperature range. In terms of the height of the internal friction peak, grain boundary damping is an important influencing factor, that is, the optimum high-temperature processing temperature of ductile iron.

In Figure 5, the maximum influencing factor of the internal friction peak as a function of temperature is presented. The vibrational energy consumed by the sliding behavior of the grain boundaries can be generally seen as the product of the relative sliding displacement between the grains and the obstruction force overcome by the grain boundary slip. When the temperature is low, although the sliding resistance between the grains is relatively large, the sliding distance along the grain boundary is minimal, and the final result is that the product of the two is not high, so the internal friction value is not high. When the temperature is high, the high-temperature causes the resistance to sliding along the grain boundary to be small, and even if the relative displacement of the sliding between the crystal grains is relatively large, the product of the two is not large, so the energy consumed during the vibration is also not high. When the temperature gradually rises from the low temperature to the appropriate temperature range, at a certain temperature in the middle, when the relative displacement between the grains and the resistance along the 
grain boundary slide are significant, the product of the two is maximized. The value also shows the peak value of the damping performance. In spheroidal graphite cast iron, in addition to the influence of temperature on the grain boundary damping mechanism, the damping mechanism and dislocation damping mechanism generated by the gap atom induced by the gap are affected by temperature.

\section{Characteristics of high-temperature heat treatment of ductile iron}

The typical ductile iron structure is composed of acicular ferrite and a retained austenite phase, both of which are carbon-containing interstitial solid solutions. In lattice structure, the former is body-centered cubic, and the latter is face-centered cubic. When the sample vibrates, the crystals will be subjected to alternating stress. The generation of lattice distortion will cause the interstitial atoms to jump back and forth, resulting in a stress-induced order phenomenon which in turn, consumes part of the vibration energy. In general, the larger the interstitial atom density of the solid solution is, the more energy is thus consumed. When the temperature is low, the diffusion of interstitial atoms has not yet proceeded, and the additional time-dependent strain is not generated, that is, the internal friction generated at this time is low. When the temperature is high, the atom diffusion rate is faster, and the additional time-dependent strain energy is generated very quickly, but at this time the phase difference between strain and stress is small, and the internal friction value is also small. When the temperature is moderate, the internal friction will reach a peak. At the same time, the temperature can change the slipping ability of the dislocation line and has influences on the damping caused by the sliding of the dislocation line. There are interactions between the dislocation line, the gap atom and the grain boundary, and various damping mechanisms. In addition to the increase in the temperature of the ductile iron sample for testing, there will be some decomposition of retained austenite which will also have impacts on the damping properties of the sample.

\subsection{Influence of test frequency on damping properties of nodular cast iron}

An ideal elastomer, when applied with stress below the elastic limit, produces a transient, fixed strain, that follows Hooke's law. However, in an actual material, when it is subjected to a stress an additional strain will appear. This additional strain is a function of time, also known as relaxation strain. The relaxation time represents the maximum time between transitions between the two equilibrium states of the material and is a parameter describing the internal friction of the material. That is to say, after applying stress at this time, only a part of the strain disappears instantaneously, and the strain of other parts gradually disappears with the extension of time, which no longer follows Hooke's law, which is the hysteresis phenomenon of the metal material. It is called dynamic hysteresis internal friction because it involves relaxation time. So, this internal friction is related to frequency.

In metal materials, there is also a static hysteresis type internal friction, which is caused by microplastic deformation inside the material. After the stress is applied and removed, the material is permanently deformed by internal microplastic deformation and the material is restored to its original state only when the reverse load is applied. When an alternating load is applied to the material, a strain ratio stress lag similar to the dynamic lag is generated, resulting in internal friction, which is instantaneously generated due to stress, so this is instantaneously generated. The static hysteresis type internal friction is not affected by the frequency but is related to the strain amplitude. The damping behavior of ductile iron comes from a variety of internal mechanisms. For the damping alloy of this composite mechanism, in addition to the influence of strain amplitude on its damping performance, the vibration frequency will also have a particular impact on its damping performance. Therefore, it is necessary to explore the influence of frequency on its damping performance.

It can be seen from Figure 6 that the damping of spheroidal graphite cast iron increases with the increase of frequency. The internal consumption value of ductile iron sample increases gradually and fluctuates slightly during the ascent. When the frequency is higher than $50 \mathrm{~Hz}$, the internal consumption value is slightly higher. The decrease in internal power consumption increases when the frequency reaches about $70 \mathrm{~Hz}$. However, as a whole, when the frequency is in the range of 1 to $100 \mathrm{~Hz}$, the higher the frequency, the higher the internal friction value of the material, from about 0.011 at low frequencies to about 0.027 at high frequencies, that is, within this frequency range. The higher the frequency, the more vibration energy 


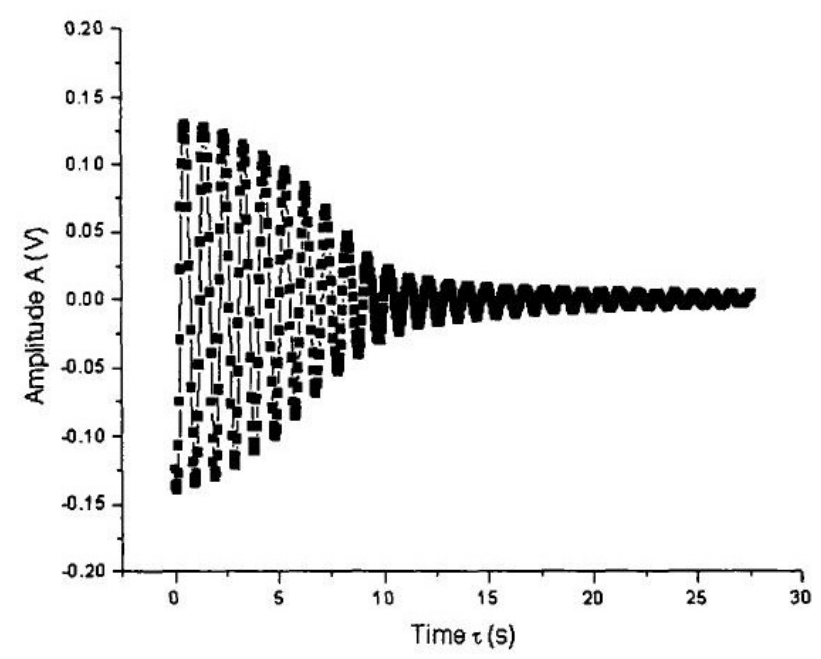

Figure 6: Damping and frequency relationship of ductile iron

the spheroidal graphite caster absorbs, indicating that the parts made of ductile iron are suitable for high-frequency vibration and can exhibit excellent damping characteristics.

\subsection{Damping effect analysis of high strength and ductile iron}

\subsubsection{Inductive ordering of interstitial atoms}

The carbon atoms in the ferrite are located at the interstitial position of the octahedron, that is, at the face center or the edge of the unit cell. The octahedral gap is located in the body center cube. Since it is asymmetrical, the carbon atoms are dissolved. This will cause distortion, and this distortion is also asymmetrical. When alternating stress acts on the sample, the carbon atoms will jump back and forth in the unit cell in order to reduce the distortion energy, which is a phenomenon of stress-induced order, and an additional strain is generated inside. The additional strain lags behind the external stress in time, so when the alternating stress acts, the back and forth jump of the carbon atoms produces the internal energy consumption, and the temperature and frequency have certain influence on it.

The carbon atoms in austenite are dissolved in the regular octahedral gap, and the distortion produced by them is symmetrical. Theoretically, when stress acts, it does not produce induced order, but if there is some apex angle on the octahedral gap, when a solvent atom is replaced by another atom or the solvent atom is removed to leave a vacancy, an asymmetrical distortion occurs, and when exter- nal stress is applied to the sample, a sense is generated. In turn, the orderly phenomenon produces the internal energy loss of the material; the greater the density of the interstitial atoms is, the higher the internal friction is.

When the temperature is low, the diffusion of interstitial atoms has not yet proceeded, and the additional time-dependent strain is not generated, that is, the internal friction generated at this time is not much. When the temperature is high, the atom diffusion rate is faster, and the time-dependent additional strain energy is generated very quickly, but at this time, the phase difference between strain and stress is small, and the internal friction value is also small. When the temperature is moderate, the internal friction will reach the peak value. At the same time, the temperature will also affect the solid solubility of the interstitial atoms. The internal consumption is a dynamic hysteresis type internal friction, and the internal friction is related to the frequency.

\subsubsection{Movement of dislocations in the basal body}

The movement of the dislocations leads to the plastic deformation of the metal. When the alternating stress acts, there will be different forms of dislocation motion, which can produce the internal friction of the dynamic hysteresis type or the static hysteresis type.

In the acicular ferrite, the carbon atoms form an air mass around the dislocations, and the dislocations are divided into a plurality of small segments by the strong nails, and the movement is performed by the bending of the separated dislocation segments. When moving in this way, it will cause the redistribution of carbon atoms in the air mass, which will hinder the movement of dislocations. The diffusion of carbon atoms in the air mass will also affect the rate of dislocation motion. The internal friction of the material, which is the dynamic hysteresis type, the higher the density of dislocations and the content of carbon atoms, the more pronounced damping behavior. When the strain amplitude is increased enough to cause the dislocation line to be unpinned, the dislocation line is still in the dislocation network, and the alternating stress acts to produce a static hysteresis type internal friction.

That is to say, when the strain amplitude is low, the dislocations are pinned by the strong pinning point, and only the small segment of the dislocation line vibration provides internal friction. At this time, the dynamic hysteresis type internal friction is generated, and as the strain amplitude increases, the dislocation line increases. It can be separated from the strong pinning point, but the dislocation line is still in the network of dislocations. When 
there is alternating stress, static hysteresis internal friction will occur.

\subsubsection{Sliding damping of ductile iron}

There are complex interactions between internal dislocations, and there are also interactions between dislocations and interstitial atoms, all of which have an effect on the motion of dislocation lines. High temperature can promote the binding of the dislocation line from the pinning point, and the frequency will also influence it. However, when the dislocation line is detached from the pinning point, the internal friction caused by the dislocation is static. Most of the atoms are irregularly arranged at the grain boundaries. The structure is amorphous, and the thickness is about several to several hundred atoms. It is affected by the purity of the metal and the phase difference between adjacent grains. The grain boundary has an absolute viscosity. When the alternating stress acts on the ductile iron material, the relative sliding on both sides of the grain boundary will occur, and the strain will lag behind the stress, which will lead to the generation of the relaxation type in ternal friction, which belongs to the dynamic hysteresis type. The grain boundary consumes the following characteristics: the vibration energy consumed by the grain boundary sliding can be approximated as the product of the displacement between the relative sliding of the grains and the blocking force overcome along the grain boundary slip. When the temperature is low, the grain boundary viscosity is strong, and the resistance of slip is considerable, but at the same time, the relative displacement of the sliding is small, so the product of the two is not high. When the temperature is high, the grain boundary viscosity is weak. The resistance of the slip becomes small, and even if the distance of the relative slip increases, the product of the two is not high. When the temperature is moderate, the product of the two reaches a maximum value, and there is an internal friction peak.

The more beautiful the crystal grains and the larger the number is, the more grain boundaries are, the larger the contact area between the crystal grains is, the stronger the damping behavior generated by the grain boundaries is, and the higher the internal friction generated inside the material is. However, the small grain size and the grain boundary will reduce the damping behavior caused by the order of the interstitial atoms. Because the grain boundary will adsorb some interstitial atoms, the higher the number of grain boundaries are, the more interstitial atoms are adsorbed, which significantly affects the gap. The diffusion of atoms reduces the internal friction caused by the diffusion of interstitial atoms. Therefore, in the case of ductile iron, a material with a complex damping mechanism, the internal mechanisms interact with each other. If there are two structurally similar phases in the tissue, the two-phase interface also has a similar damping behavior as above.

\subsubsection{Ferromagnetic damping analysis of acicular ferrite}

Ferrite is magnetic. When there is alternating stress, it changes its magnetization state, resulting in additional strain. At this time, the phenomenon that the strain lags behind the stress occurs and a part of the vibration energy is consumed. There are three main types of such magnetoelastic internal friction. The first type is the internal friction generated by macroscopic eddy currents; when the stress acts on the partially magnetized sample the sudden change in magnetization causes the eddy current to be induced on the surface of the sample and the diffusion of the eddy current causes the material to be magnetic. The stretching effect creates additional strain, causing the strain to lag behind the stress, which in turn produces damping internal friction. The second type is the internal damping friction generated by microscopic eddy currents: when the strain acts on the demagnetizing material, although the macroscopic eddy current is not generated, microscopic eddy currents are generated in the microscopic domain size region and the relaxation type internal friction is generated. The third type is static hysteresis type internal friction; the action of stress causes the magnetic domain wall inside the material to irreversibly move and only recovers when the stress is reversely loaded. There-

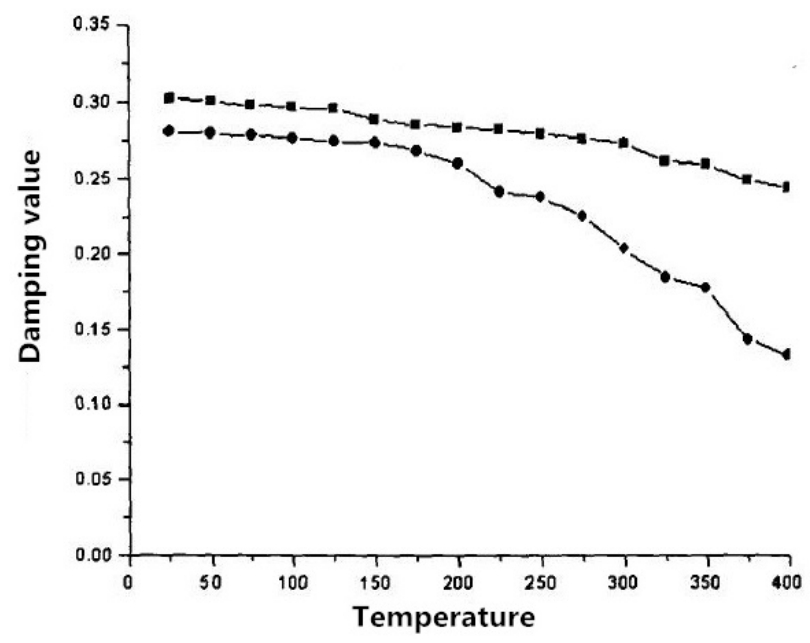

Figure 7: Damping analysis of ductile iron 
fore, when the alternating stress acts, static hysteresis type internal friction occurs as can be seen from Figure 7 .

\subsubsection{Thermoelastic damping}

Metal materials expand when heated. Conversely, under adiabatic conditions, when stress acts on the metal material, heat is inevitably generated inside the metal material. This heat flow causes additional strain inside the material. In turn, the loss of energy inside the metal material is caused by the dynamic hysteresis type. If the frequency of stress is high, the heat is not exchanged inside the material, and no heat is generated at this time. If the frequency of the stress is low, there is sufficient time for heat exchange inside the metal material in each cycle and the internal temperature is at each point. In the equilibrium state, no internal friction is generated at this time. When the measurement frequency of the sample is lower than $100 \mathrm{~Hz}$, the influence of such damping behavior may not be considered. In summary, the ductile iron is composed of acicular ferrite, retained austenite and graphite balls. This complex phase damping alloy interacts with various damping mechanisms during vibration, and its internal friction is the result of the interaction of various mechanisms. High-temperature grading heat treatment dramatically enhances the strength of the alloy but the plasticity decreases. Therefore, the high-temperature heat treatment can dramatically increase the hardness of the alloy. Grain refinement also contributes to the hardness of the alloy.

\section{Conclusion}

The treated ductile iron has a high toughness ferrite matrix in addition to spheroidal graphite and a small amount of agglomerated graphite. It eliminates free cementite in the as-cast structure and has a uniform, fine short-needle martensite with a small amount of retained austenite in the structure. The tempered sorbite structure is evenly distributed, and the damping performance is improved. The damping reduction of ductile iron during heat treatment mainly comes from the high-temperature heat treatment process of the workpiece. The high-temperature treatment can improve the performance of ductile iron to a certain extent.

\section{References}

[1] Lin C.K., Hung T.P., Influence of microstructure on the fatigue properties of austempered ductile irons-low-cycle fatigue, nt. J. Fatigue, 1996, 18, 309-320.

[2] Jincheng L., Guoxiong S., Impact toughness and fracture toughness of austempered ductile iron, CHINA FOUNDRY, 2004,1,2,7988.

[3] Putatunda S.K., Development of austempered ductile cast iron with simultaneous high yield strength and fracture toughness by a novel two step austempering process, Materials Science and Engineering, 2001, 315, 70-80.

[4] Sohi M.H., The role of austempering parameters on the structure and mechanical properties of heavy section ADI, Journal of Materials Processing Technology, 2004, 153-154.

[5] Carvalho M. A. D., Xavier R.R., Filho C.S.P., et al., Microstructure, mechanical properties and wear resistance of high speed steel rolls for hot rolling mills, 2002, 29, 27-32.

[6] Tanaka T., Hashimoto M., Koie T., et al., High speed steel type cold rolling mill roll by continuous pouring process for cladding, Nippon Steel Technical Report, 2002, 86, 80-85.

[7] Baker H., ASM Handbook, Volume 3, Alloy Phase Diagrams, ASM International, Materials Park, Ohio, 1992, 281.

[8] Ambroza P., Kavaliauskiene L., Influence of carbon and boron on the properties of deposited layer, Mechanika, 2004, 4, 59-63.

[9] Changqing G., Improving ductility and toughness of Fe-C-B cast iron, Australia: The University of Queensland, 2002.

[10] Han J.M., Zhou Q., Bar G.C., Study of the effects of austempering temperature and time on scuflng behavior of austempered $\mathrm{Ni}-\mathrm{Cu}$ ductile iron,. 2012, 2, 99-105.

[11] Granct A., Theory of mechanical damping due to dislocation. Journal of Applied Physics, 1956, 276, 583-593.

[12] Noral A., Advances in the metallurgy and applications of ADI, Journal of Metallurgical Engineering, 2013, 2, 1-18.

[13] Zamts A., Msdff M., Grese M., Influence of shot peening on the fatigue life of $\mathrm{Cu}-\mathrm{Ni}$ austempered ductile iron, Materials Science and Engineering, 2012, 545, 78-85.

[14] Richard H.L., James J.K., Study on Damping Characteristics of Metal Cast Iron, Natural Science, 2014, 6, 127-129.

[15] Ritchie I.G., Pan Z.L., High Damping Metals and Alloys, Metall. Trans, 1991, 22A, 607-616.

[16] Ritchie I.G., Pan Z.L., Goodwin F. E., Characterization of the Damping Properties of Die Cast Zinc-Aluminum Alloys, Metall. Trans, 1991, 22A, 617-622.

[17] James D.W., High Damping Metals for Engineering Application, Mater Sci Eng,. 1969, 4, 1-8.

[18] Zhang R.J., Perez Lavernia E.J., Documentation of Damping Capacity of Metallic, Ceramic and Metal-Matrix Composite Materials, J. Mater. Sci, 1993, 28, 9, 2395-2404. 